\title{
Dissolution Kinetics in Chemically Amplified EUV Resist
}

\author{
Hiroki Yamamoto ${ }^{a^{*}}$, Takahiro Kozawa ${ }^{a}$, Seiichi Tagawa ${ }^{a}$, \\ Takeyoshi Mimura $^{\text {b, }}$, Takeshi Iwai ${ }^{b}$, and Junichi Onodera ${ }^{\text {b }}$ \\ ${ }^{\mathrm{a}}$ The Institute of Scientific and Industrial Research, Osaka University, \\ 8-1 Mihogaoka, Ibaraki, Osaka 567-0047, Japan \\ ${ }^{\mathrm{b}}$ Tokyo Ohka Kogyo Co., Ltd., \\ 1590 Tabata, Samukawa, Koza, Kanagawa 253-0114, Japan, \\ *E-mail address: hiroki@sanken.osaka-u.ac.jp
}

\begin{abstract}
Polymer structure effects on the dissolution kinetics and deprotection reaction were investigated to understand inherent extreme ultraviolet (EUV) resist characteristics. The effect of protecting groups and protecting ratio on activation energy were observed. Also, the protecting ratio effect on dissolution kinetics was observed. The speed of TMAH penetration into each resist film was different for each resist as the concept of the "penetration zone". This indicated that the dominant rate-determining step in resists is TMAH penetration into the resist film. Thus, the dissolution rates were affected by changing the protection ratio of polymer. Moreover, the preliminary evaluation of resist profile for EUV chemically amplified resists was performed by using PROLITH and parameters obtained with EUV exposure tool. Furthermore, the resist patterns were produced by electron beam and the SEM observation results were compared with the PROLITH simulation results. It is important for EUV lithography to select appropriate protecting group and protecting ratio.
\end{abstract}

Keywords: EUV lithography, chemically amplified resist, dissolution kinetics, deprotection reaction,

\section{Introduction}

Beyond the $16 \mathrm{~nm}$ node, extreme ultraviolet (EUV) lithography is regarded as the most promising Next Generation Lithography (NGL) technology. In ITRS 2008 road map, the target of resist performance in EUV lithography is set to be as following. Resolution, line width roughness (LWR) and sensitivity requirements for DRAM half pitch are $16 \mathrm{~nm}, 0.9 \mathrm{~nm} 3 \sigma, 10 \mathrm{~mJ} / \mathrm{cm}^{2}$, respectively.[1] Thus, the requirement of resist performance individually has become very strict demand. Moreover, it becomes the most difficult task to simultaneously fulfill these requirements because there is a trade-off among these requirements.[2] In order to overcome these problems concurrently, it is very important to separately understand reaction mechanisms of the resist pattern formation and inherent resist characteristics such as acid generation efficiency, dissolution kinetics, deprotection reaction and so on.

In pattern formation of chemically amplified resists, the energy is first deposited on resist materials by incident radiation such as EUV radiation. Using the accumulated energy, acids are generated. The acids catalyze pattern formation reactions during PEB via typically the deprotection reaction of polymers, causing the change in alkaline solubility of the resist in exposed areas.[3] It is indispensable for feasibility of EUV lithography to grasp basic chemistry of resist matrices in all stage.

So far, we have estimated acid generation efficiencies in resist matrices and elucidated the reaction mechanism of chemically amplified resist for ionizing radiation such as Electron beam (EB) 
and EUV. [4-6, 9] The acid generation mechanism for EUV resists is analogous to that of EB resists. [7-9] However, the absorption of incident EUV photon conforms to Lambert's Law in EUV resists as well as $\mathrm{KrF}$ and $\mathrm{ArF}$ photoresists. Thus, it is clarified that EUV resists have some similarity to those in both photoresists and EB resists.

With the reduction in feature sizes, the acid generation efficiency and chemical reactions such as deprotection reaction and dissolution kinetics in resist films becomes critical to accomplish high resolution and high sensitivity. Especially, dissolution kinetics and deprotection reaction of resist films is a key factor to pattern formation. Therefore, it is important to understand dissolution kinetics and deprotection reaction of resist films for the feasibility of EUV lithography.

Because the acids are generated by the ionization of polymer in EUV resists, acid generators hardly affect the absorption coefficient of EUV.[10, 11] Thus, polymers have to be modified in order to adjust the absorption coefficient of EUV resists. Therefore it is essential to investigate polymer structure effects on the dissolution kinetics and deprotection reaction. In this study, polymer structure effects on the dissolution kinetics and deprotection reaction were investigated using a resist development analyzer (RDA-800EUV)[12-13] measurement system (Litho Tech Japan) and deprotection reaction analyzer. [14] The time dependent reactions of the acid-labile protecting group was monitored by FT-IR and the kinetic parameters such as deprotection reaction constants and activation energies were obtained from these data. Also, the simulation of resist profiles for EUV chemically amplified resists was performed by calculation using PROLITH and parameters obtained with EUV exposure tool. Furthermore, the resist patterns were produced by EB and the SEM observation results were compared with the PROLITH simulation results.

\section{Experimental}

In experiments, PHS derivatives partially-protected by three kinds of protecting groups were used as a polymer (Polymer A C) as shown in Figure 1. Triphenylsulfonium-triflate (TPS-tf) was used as an acid generator. Propylene glycol monomethyl ether acetate (PGMEA) was used as a casting solvent. Resist film thickness was measured with a film thickness measurement system (Filmetrics F20, spectroscopic

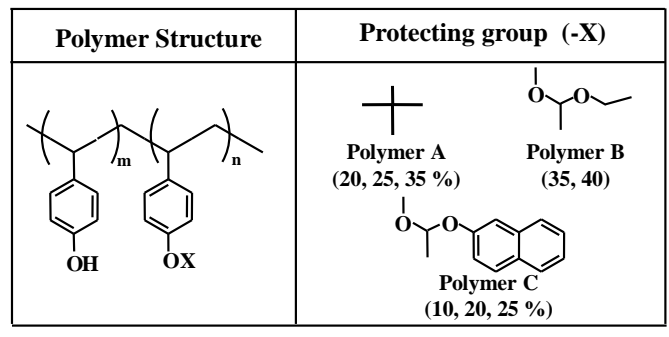

Fig. 1. Polymer structure.

ellipsometer) and a Dektak3ST surface profiler.

In the experiments for the analysis of deprotection reaction, resist solutions were spin-coated onto silicon substrates and were pre-baked under the conditions of dissolution kinetics measurements. Then the films were exposed to EUV radiation (Energetic, EQ-10M). The exposed area was approximately $1 \times 1 \mathrm{~cm}^{2}$. The exposure doses ranged from 0.1 to $1 \mathrm{~mJ} / \mathrm{cm}^{2}$. After the exposure, they were baked in the temperature range of $80-140{ }^{\circ} \mathrm{C}$ for $150 \mathrm{~s}$ to estimate deprotection reaction. We used deprotection reaction analyzer to analyze deprotection reactions for EUV lithography. This system makes it possible to measure thin film by using an MCT detector that offered higher detection sensitivity. IR measurements were performed with a resolution of $4 \mathrm{~cm}^{-1}$ over the wave number range from 700 to $4000 \mathrm{~cm}^{-1}$. Typically 64 spectra were averaged from each exposure measurement and single measurement scan was performed with sampling every ca. 0.9 second. By analyzing subtractive spectra between spectrum obtained in resist films and spectrum of the only $\mathrm{Si}$ wafer, we can extract information on the resist such as deprotection reaction constant (Kdp), the activation energy of deprotection reactions in resist matrices and so on.

In the sample preparation for dissolution kinetics measurements, resist solutions containing $3.1 \mathrm{~mol} \%$ acid generator were spin-coated onto silicon substrates at $3000 \mathrm{rpm}$ for $30 \mathrm{~s}$ to form thin films. After the exposure, dissolution rate measurements were conducted at $110{ }^{\circ} \mathrm{C}$ for $60 \mathrm{~s}$ under PEB conditions within $1 \mathrm{~min}$ of intial exposure to minimize airborne contamination. They were developed in NMD-3 developer [2.38 \% tetramethylammonium hydroxide (TMAH) solutions] for $30 \mathrm{~s}$ at $23{ }^{\circ} \mathrm{C}$ and the dissolution kinetics was investigated using a resist development analyzer (RDA) measurement system (Litho Tech Japan). For this analysis tool, the resist dissolution is obtained by measuring the thickness of resist films coated on $\mathrm{Si}$ before and after 
development. The dissolution rate was estimated by monitoring the variation in interference of a 470 $\mathrm{nm}$ wavelength light reflected at the $\mathrm{Si}$ substrate and resist film surface as resist film dissolve in TMAH developer.

Also, EUV lithography simulation was performed by using these data obtained with EUV exposure tools. Furthermore, the resist patterns were produced by $75 \mathrm{kV}$ electron beam. The exposure doses ranged from $25 \mu \mathrm{C} / \mathrm{cm}^{2}$ to $50 \mu \mathrm{C} / \mathrm{cm}^{2}$. After the exposure, they were baked in the temperature range of $80-140{ }^{\circ} \mathrm{C}$ for $60 \mathrm{~s}$. They were immersion-developed in $2.38 \mathrm{wt} \%$ TMAH solutions for 30 s and rinsed in water before drying. Resist patterns were recorded with a SEM (JSM-6335F, JEOL).

\section{Results and discussion}

FT-IR spectroscopy can directly measure changes in the photoactive species by measuring absorbance peaks unique to the photoactive species. Figure 2 shows the representative results of in situ

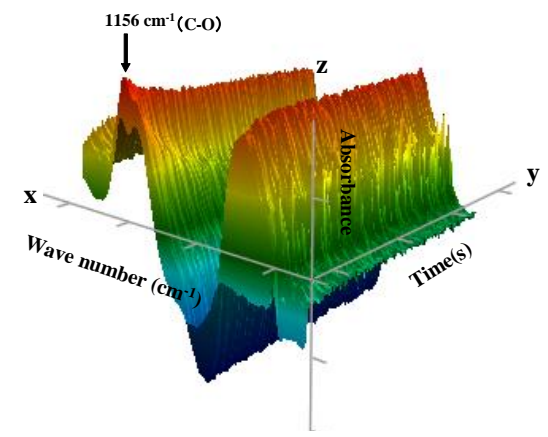

Fig. 2. FT-IR real-time measurements of Polymer A during PEB after the expose to EUV. The $\mathrm{x}, \mathrm{y}$ and $\mathrm{z}$ axis represents the wave number $\left(\mathrm{cm}^{-1}\right)$, PEB time (s), the absorbance, respectively. The change in the 1156 $\mathrm{cm}^{-1}$ absorption peak of ester (C-O) bonds accompanying deprotection reaction.

FT-IR measurements of Polymer A during PEB after the expose to EUV. The $\mathrm{x}, \mathrm{y}$ and $\mathrm{z}$ axis represents the wave number $\left(\mathrm{cm}^{-1}\right)$, PEB time (s), the absorbance, respectively. As shown in Figure 2, the deprotection reactions in polymer $\mathrm{A}$ were observed based on the changes in the $1156 \mathrm{~cm}^{-1}$ absorption peak of ester (C-O) bonds accompanying deprotection reaction. With the PEB time elapse, the decrease in the ester bond absorption peak can be seen in Figure 2. Also, we examined the changes in the $\mathrm{C}-\mathrm{O}$ bond spectra near $1156 \mathrm{~cm}^{-1}$ without the exposure to EUV to investigate whether additional chemical reaction during PEB was occurred. Subtractive spectra weren't changed in the $1156 \mathrm{~cm}^{-1}$ region. These represent the deprotection reaction proceeds rapidly during PEB after EUV exposure. In the case of Polymer B and C, the changes in the 1230 $\mathrm{cm}^{-1}$ absorption peak of antisymmetric stretching motion (C-O-C) bonds accompanying deprotection reaction and that in the $947 \mathrm{~cm}^{-1}$ absorption peak were observed. However, the changes in the 1230 $\mathrm{cm}^{-1}$ absorption peak was observed during PEB without EUV exposure while it wasn't observed at the $947 \mathrm{~cm}^{-1}$ absorption peak. So, the change in the $947 \mathrm{~cm}^{-1}$ absorption peak was monitored as previously reported.[15-17]

In order to analyze the kinetics of the deprotection reaction, the change in IR absorbance with exposure time was converted into a normalized protection group concentration. The relative concentration of protected sites was then measured as a function of bake time for many exposure energies and PEB temperatures. By appling a newly deprotection reaction model[18] (equation (1) below) revised with Petersen's and Ohfuji's deprotection reaction model[19,20] to obtained results, best fit values for the deprotection reaction rate constant $\mathrm{Kdp}$ could be determined.

$$
\begin{aligned}
& \left.P=\left\{\frac{1}{1+\left(\frac{E}{A}\right)^{b}}\right\} \exp \left\langle K_{d p} \bullet t \bullet[11-\exp (-C E)\} \bullet \exp \left(-K_{\text {loss }} \bullet t\right)\right]^{m}\right\rangle \\
& K_{d p}=-\frac{K_{a m p} \bullet K_{d i f-c o n t} \bullet D}{K_{a m p}+K_{d i f-c o n t} \bullet D} \quad \ldots \ldots
\end{aligned}
$$

Where $[\mathrm{P}]$ is the relative concentration of protecting group, $\mathrm{Kdp}$ is the deprotection reaction constant during PEB $\left(\mathrm{s}^{-1}\right), \mathrm{m}$ is the deprotection reaction order in PEB, E is the exposure energy,

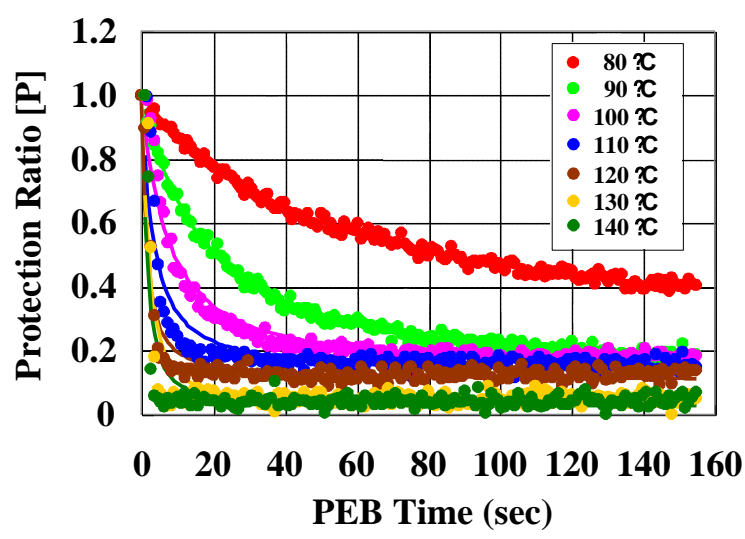

Fig. 3. Relationship between deprotection ratio and $\mathrm{PEB}$ times in the temperature range of $80-140{ }^{\circ} \mathrm{C}$ for Polymer A which is protected by $35 \%$ protecting groups. Circles and lines indicate the measured values and the results of fitting to the equation of (1), respectively. 
and $t$ is PEB time, $T_{d}$ is the reaction delay constant in PEB and $\tau_{1}$ is the average acid lifetime constant in PEB.

Figure 3 shows the relationship between deprotection rate and PEB times, called the deprotection reaction curves, in the temperature range of $80-140{ }^{\circ} \mathrm{C}$ for Polymer $\mathrm{A}$, which is protected by $35 \%$ protecting groups. The exposure dose was $1.4 \mathrm{~mJ} / \mathrm{cm}^{2}$. Circles and lines indicate the measured values and the results of fitting to the equation, respectively. As seen in Figure 3, good fits to data were obtained. The deprotection reaction curves in Polymer $\mathrm{B}$ and $\mathrm{C}$ were also obtained by the same way as polymer A. Based on the deprotection curves shown in Figure 3, deprotection reaction constant (Kdp) and the activation energy of deprotection reactions in Polymer A, B, and C were calculated. Arrhenius plots were made by these Kdp in order to estimate the activation energies. Figure 4 shows Arrhenius

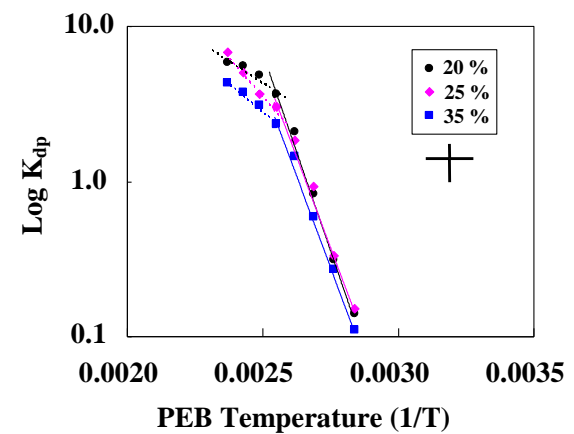

Fig.4 The activation energies of Polymer A.

plots of Polymer A. As seen Figure 4, Arrhenius plots have different two regions. The Arrhenius plots were composed of different two regions. One is the regions at low temperature where the reaction rate is controlled by the deprotection reaction. The other is that at high temperature where the reaction rate is controlled by acid diffusion. These agreed with the Byers and Petersen model.[24, 25]

The activation energy constants are summarized in Table 1.The variation of Kdp with

Table 1. The activation energies in three kinds of polymers, Polymer A C..

\begin{tabular}{|c|c|c|c|}
\hline Polymer A & $(20 \%)$ & $(25 \%)$ & $(35 \%)$ \\
\hline $\begin{array}{c}\text { Activation energy } \\
\text { (kcal/mol) }\end{array}$ & 21.4 & 38.3 & 27.8 \\
\hline Polymer B & $(40 \%)$ & $(45 \%)$ & \\
\hline $\begin{array}{c}\text { Activation energy } \\
\text { (kcal/mol) }\end{array}$ & 3.34 & 8.61 & \\
\hline Polymer C & $(10 \%)$ & $(20 \%)$ & $(25 \%)$ \\
\hline $\begin{array}{c}\text { Activation energy } \\
\text { (kcal/mol) }\end{array}$ & 1.16 & 1.16 & 3.20 \\
\hline
\end{tabular}

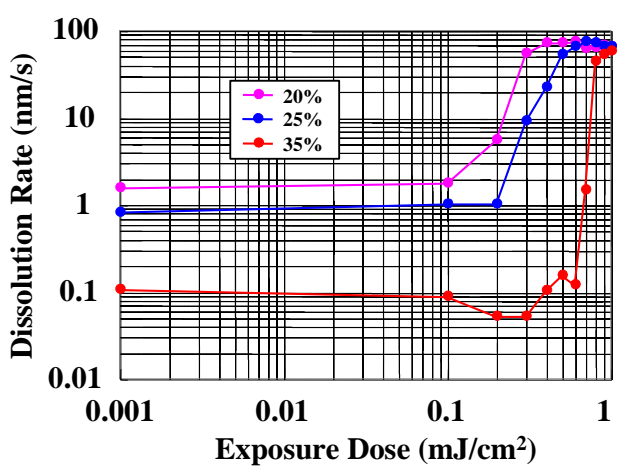

Fig. 5 Dissolution rate curve according to protection ratio of Polymer $\mathrm{A}$. The curves relate the resist dissolution rate as a function of exposure dose on a $\log / \log$ scale.

temperature follows a classical Arrhenius behavior with the activation energy of ca.21.4-27.8 $\mathrm{Kcal} / \mathrm{mol}$ for polymer A. Though the value obtained was similar to the value reported for DUV, $[18,21]$ it is less than Ea of $32 \mathrm{kcal} / \mathrm{mol}$ value reported by Long et al and J. Hutchinson.[22,23] This might be due to the difference of acid generator used in experimental. For the small protecting groups such as Polymer A and Polymer $\mathrm{B}$, the dependence of activation energy on protecting ratio was small. On the other hand, for bulky protection group, the protecting ratio significantly affected the activation energy probably due to the effect of steric hindrance. Thus, the effect of protecting groups and protecting ratio on activation energy was observed.

Also, the effect of protecting group and protecting ratio on dissolution kinetics was investigated. Figure 5 shows the dissolution rate curve according to protection ratio of Polymer A. The curves relate the resist dissolution rate as a function of exposure dose on a $\log / \log$ scale. The dissolution curve obtained is similar to those reported elsewhere. [26-29] The function of exposure dose on a $\log / \log$ scale have an $\mathrm{S}$ shape as shown in Figure 5. This implied that the dissolution rate change was very small in the low dose region, then abruptly increased at threshold dose, and finally converged to a constant rate. The deterioration of sensitivity was observed with the increase of protecting ratio while the dissolution slope increases with increase of protecting ratio.

The dissolution parameters such as maximum dissolution rate (Rmax) and minimum dissolution rate (Rmin) were obtained from Figure 5. A slight difference in Rmax was observed due to the difference of resulting products. Also, Rmax at each protecting ratio did not have a significant large difference. On the other hand, Rmin 
decreased with increase of protecting ratio. Higher protect ratio provided increased inhibition. A large value of Rmin signifies that the resist easily and smoothly dissolves into alkaline developer. These results suggest that a higher protecting ratio increase the resists potential for higher resolution capability as previously reported. [26, 30] It is reported that the dissolution of resists to an alkaline developer is attributed to the penetration of base molecules from the developer into the resist by base molecule diffusion through the hydrophilic sites of the polymers. This is called as the concept of the "penetration zone". [31-33] The speed of penetration increases with the increasing volume of the hydrophilic space of the resist film. On the other hand, because hydoroxy group of base resin is hydrophilic TMAH solution, it can easily penetrate into resist film as reported. [27, 28, 34] Thus, it is supposed that the hydrophilic substituent content of the base polymer determines the degree of penetrability of the base molecules and the dissolution rate of the resist to the developer. The speed of TMAH penetration into each resist film is different for each resist in our study. This indicated that the dominant rate-determining step in these resists is TMAH penetration into the resist film.[35] The dissolution rates was affected by changing the protection ratio of polymer.

Furthermore, we performed EUV lithography simulation by using these data obtained with EUV exposure tools. The calculation parameters of Polymer on PROLITH are shown in Table 2. The partially coherence factor of the illumination system was 0.5 . The resist line width was set to 26 $\mathrm{nm}$ and $50 \mathrm{~nm}$ 1:1 line\& space pattern. The exposure dose is set to $20,25,30 \mathrm{~mJ} / \mathrm{cm}^{2}$. At the film thickness of $50 \mathrm{~nm}$, an almost vertical is obtained for Polymer A. On the other hands, at the film thickness of $26 \mathrm{~nm}$ there is considerable bridge of pattern side walls, and good quality patterns were not obtained as shown in Figure 6.

Table 2. Calculation parameters of Polymer A on PROLITH.

\begin{tabular}{|c|c|}
\hline \multicolumn{2}{|c|}{ Development Parameters } \\
\hline Development Rmax & $68.6 \mathrm{~nm} / \mathrm{s}$ \\
\hline Development Rmin & $0.10 \mathrm{~nm} / \mathrm{s}$ \\
\hline Development Mth & 0.39 \\
\hline Development n & 18.2 \\
\hline \hline \multicolumn{2}{|c|}{ PEB Parameters } \\
\hline Thermal Decomp. Ea & $27.8 \mathrm{~kJ} / \mathrm{mol}$ \\
\hline Thermal Decomp. $\ln ($ Ar) & $6.1 / \mathrm{s}$ \\
\hline \multicolumn{2}{|c|}{ ABC Parameters } \\
\hline A & $0 / \mu \mathrm{m}$ \\
\hline B & $1.06 / \mu \mathrm{m}$ \\
\hline C & $0.08997 \mathrm{~cm} / \mathrm{mJ}$ \\
\hline
\end{tabular}

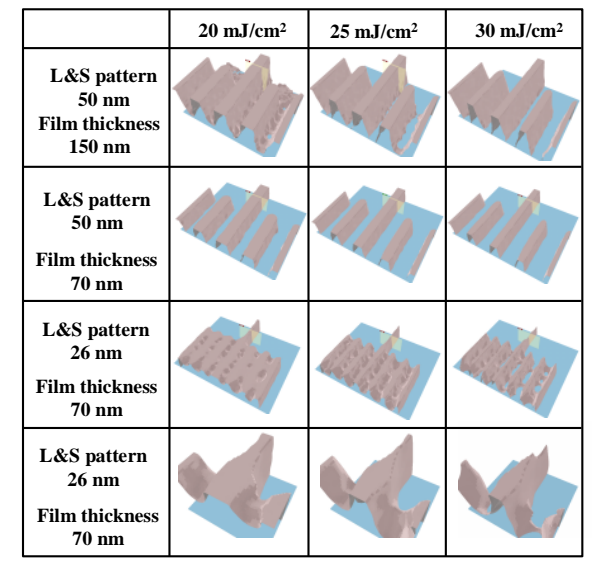

Fig. $650 \mathrm{~nm}$ and $26 \mathrm{~nm}$ L\&S pattern profiles of Polymer A resist, calculated by PROLITH.

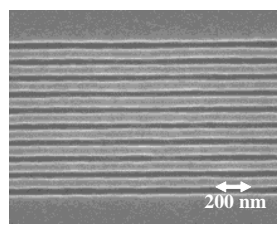

Fig. 7 SEM image of L\&S pattern in Polymer A resist by using $75 \mathrm{kV}$ EB.

The resist patterns were produced by EB and SEM observation result was compared with PROLITH simulation result. Figure 7 shows representative SEM images of Polymer A. As for the resolution, the resolution limit by using EB is almost same as prediction based on PROLITH. PROLITH might suggest the possibility of rough examination of resolution limits for EUV resist.

\section{Conclusion}

Polymer structure effects on the dissolution kinetics and deprotection reaction were investigated to understand inherent EUV resist characteristics. The effect of protecting ratio on activation energy was observed. For small protecting group, dependence of activation energy on protecting ratio was small. On the other hands, for bulky protecting group protecting ratio significantly affect the activation energy probably due to the effect of steric hindrance. Also, the deterioration of resist sensitivity was observed with increase of protecting ratio while the dissolution slope increases with increase of protecting ratio. Thus, the dissolution rates and sensitivity were more affected by changing the protection ratio of polymer than the type of protecting group. Also, we performed EUV lithography simulation by using PROLITH. It is important for EUV lithography to select appropriate protecting group and protecting ratio. 


\section{Acknowledgement}

This work was supported in part by a Grant-in-Aid for Scientific Research (Project No. 21760584) from the Ministry of Education, Culture, Sports, Science and Technology of Japan (MEXT).

The authors wish to thank Dr. Atsushi Sekiguchi and Dr. Mariko Isono of Litho Tech Japan co. jp for useful advice as for PROLITH.

\section{References}

1. International Technology Roadmap for Semiconductors 2008 update.

http://www.itrs.net/Links/2008ITRS/Update/200 8Tables_FOCUS_B.xls

2. G. M. Gallatin, Proc. SPIE 5754, 38 (2005).

3. H. Ito, Adv. Polym. Sci. 172, 37 (2005)

4. H. Yamamoto, A. Nakano, K. Okamoto, T. Kozawa, and S. Tagawa, Jpn. J. Appl. Phys. Part 1 43, 3971 (2004).

5. H. Yamamoto, T. Kozawa, A. Nakano, K. Okamoto, S. Tagawa, T. Ando, M. Sato, and H. Komano, J. Vac. Sci. Technol. B 23, 2728 (2005).

6. H. Yamamoto, T. Kozawa, A. Nakano, K. Okamoto, S. Tagawa, T. Ando, M. Sato, and H. Komano, Jpn. J. Appl. Phys. Part 1 44, 5836 (2005).

7. T. Kozawa, Y. Yoshida, M. Uesaka, and S. Tagawa, Jpn. J. Appl. Phys. Part 1 31, 4301 (1992).

8. T. Kozawa, S. Tagawa, H. Oizumi, and I. Nishiyama, J. Vac. Sci. Technol. B24, L27 (2006).

9. H. Yamamoto, T. Kozawa, S. Tagawa, H. B. Cao, H. Deng, and M. J. Leeson, Jpn. J. Appl. Phys. 46, L142 (2007).

10. P.Dentinger, G. Cardinale, C. Henderson, A. Fisher, and A. Ray-Chaudihuri, Proc. SPIE 3997, 588 (2000).

11. R. Hirose, T. Kozawa, S. Tagawa, T. Kai, and T. Shimokawa, Jpn. J. Appl. Phys. 46, L979 (2007).

12. A. Sekiguchi, C. A. Mack, Y. Minami and T. Matsuzawa, Proc. SPIE 2725, 49 (1996).

13. A. Sekiguchi, Y. Kono, M. Kadoi, Y. Minami, T. Kozawa, S. Tagawa, D. Gustafson and P. Blackborow, Proc. SPIE 6519, 651946-1 (1996).

14. A. Sekiguchi, Y. Kono, Proc. SPIE 6923, 69232C-1 (2008).

15. S. Nagahara, L. Yuan, W. J. Poppe, A. Neureuther, Y. Kono, A. Sekiguchi, K. Fujiwara, T. Watanabe, K. Taira, S. Kusumoto, T. Nakano and T. Shimokawa, Proc. SPIE 5753, 338 (2005).

16. Y. Miyake, M. Isono and A. Sekiguchi, Proc. SPIE 4345, 1001 (2001).

17. M. F. Diniz, A. M. Kawamoto, R. C. L. Dutra, T. Keicher, Polímeros: Coémcoa e Tecnologia 17, 46 (2007)

18. A. Sekiguchi, Y. Miyake and M. Isono, Jpn. J. Appl. Phys., Part 1 39, 1392 (2000).

19. J. S. Petersen, C. A. Mack, J. W. Thackeray, R. Sinta, T. H. Fedynyshyn, J. M. Mori, J. D. Byers and D. A. Miller Proc. SPIE 2438, 153 (1995).

20. T. Ohfuji, K. Nakano, K. Maeda and E. Hasegawa, J. Vac. Sci. Technol. B13, 3022 (1995).

21. A. Sekiguchi, C. A. Mack, M. Isono and T. Matsuzawa, Proc. SPIE 3678, 985 (1999).

22. T. Long, S. K. Obendorf and F. Rodriguez, Polymer. Eng. Sci., 32, 1589 (1992).

23. J. Hutchinson, W. Hinsberg, F. Houle, P. Seidel, R. Johnson and W. Oldham, J. Vac. Sci. Technol. B12, 3857 (1994).

24. J. S. Petersen, C. A. Mack, J. W. Thackeray, R. Sinta, T. H. Fedynyshyn, J. M. Mori, J. D. Byers and D. A. Miller Proc. SPIE 2438, 153 (1995).

25. J. S. Petersen, C. A. Mark, J. Sturtevant, J. D. Byers and D. A. Miller, Proc. SPIE 2438, 167 (1995).

26. T. Itani, K. Kaneyama, T. Kozawa and S. Tagawa, J. Vac. Sci. Technol. B26, 2261 (2008).

27. T. Itani, H. Iwasaki, H. Yoshino, M. Fujimoto and K. Kasama, Proc. SPIE 2195, 126 (1994).

28. T. Itani, H. Iwasaki, H. Yoshino, M. Fujimoto and K. Kasama, Proc. SPIE 2438, 191 (1995).

29. H. Ito, IBM J. Res. Develop. 45683 (2001).

30. M. Toriumi, K Kaneyama, S. Kobayashi and T. Itani, Proc. SPIE 6923, 69230L (2008).

31. J. P. Huang. T. K. Kwei and A. Reiser, Macromolecules 22, 4106 (1989).

32. A. Reiser, H. Y. Shih, T. F. Yeh and J. P. Huang, Angew. Chem. Int. Ed. Engl. 35, 2429 (1996).

33. Y. Zhu, L. Capodieci and F. Cerrina, Proc. SPIE 3049, 201 (1997).

34. T. Itani, H. Yoshino, S. Hashimoto, M. Yamana, M. Miyasaka and H. Tanabe, J. Vac. Sci. Technol. B16, 3726 (1998).

35. T. Inani, H. Iwasaki, M. Fujimoto and $\mathrm{K}$. Kasama, Jpn. J. Appl. Phys., Part 1 33, 7005 (1994). 